Artículo científico

(Original paper)

\title{
NUEVO REGISTRO DE SINANTROPÍA DE FILISTATOIDES INSIGNIS (ARANEAE: FILISTATIDAE) EN MÉXICO Y ACTUALIZACIÓN DEL LISTADO DE ARAÑAS ACTUALES DE CHIAPAS
}

\section{NEW SYNANTHROPY RECORD OF FILISTATOIDES INSIGNIS (ARANEAE: FILISTATIDAE) IN MEXICO AND UPDATE OF THE EXTANT SPIDERS LIST FROM CHIAPAS}

\author{
MIGUEL ÁNGEL GARCÍA VILLAFUERTE ${ }^{1 *}$, ANTONIO DOMINGOS BRESCOVIT ${ }^{2}$ \\ ${ }^{1}$ Museo de Paleontología "Eliseo Palacios Aguilera". Dirección de Paleontología. Secretaría de Medio Ambiente e Historia \\ Natural. Calzada de los hombres Ilustres s/n. C.P. 29000. Antiguo Parque Madero Tuxtla Gutiérrez, Chiapas, México. \\ <mgarciavillafuerte@yahoo.com.mx> \\ ${ }^{2}$ Laboratório Especial de Coleções Zoológicas. Instituto Butantan, Avenida Dr. Vital Brasil, 1500 CEP 05503-900 - Butantã - \\ São Paulo, Brasil. <antonio.brescovit@butantan.gov.br> \\ *Autor de correspondencia: <mgarciavillafuerte@yahoo.com.mx>
}

Recibido: 07/12/2017; aceptado: 09/07/2019; publicado en línea: 11/07/2019

Editor responsable: Guillermo Ibarra Núñez

García-Villafuerte, M. Á., Brescovit A. D. (2019) Nuevo registro de sinantropía de Filistatoides insignis (Araneae: Filistatidae) en México y actualización del listado de arañas actuales de Chiapas. Acta Zoológica Mexicana (nueva serie), 35, 1-8. https://doi.org/10.21829/azm.2019.3501136

RESUMEN. Con el descubrimiento de Filistatoides insignis (O. Pickard-Cambridge, 1896), un nuevo registro de araña sinantrópica para México, el presente estudio tiene como objetivo actualizar el listado de arañas actuales de Chiapas. Los especímenes fueron colectados en el Museo de Paleontología "Eliseo Palacios Aguilera", ubicado en la ciudad de Tuxtla Gutiérrez. Con 533 especies de arañas actuales, Chiapas alberga el $22.2 \%$ de la biodiversidad total de arañas de México, ocupando el primer lugar en la riqueza de arañas para el país.

Palabras clave: Tuxtla Gutiérrez; diversidad; biogeografía

García-Villafuerte M. A., Brescovit A. D. (2019) New synanthropy record of Filistatoides insignis (Araneae: Filistatidae) in Mexico and update of the extant spiders list from Chiapas. Acta Zoológica Mexicana (nueva serie), 35, 1-8. https://doi.org/10.21829/azm.2019.3501136

\begin{abstract}
With the discovery of Filistatoides insignis (O. Pickard-Cambridge, 1896), a new record of synanthropic spider from Mexico, this work aims to update the list of the extant spiders from Chiapas. The specimens were collected at the Museum of Paleontology "Eliseo Palacios Aguilera", located at Tuxtla Gutiérrez city. With 533 species, Chiapas houses $22.2 \%$ of the biodiversity of spiders recorded from Mexico, occupying the first place on the spider's richnnes for the country.
\end{abstract}

Key words: Tuxtla Gutiérrez; diversity; biogeography 
García-Villafuerte \& Brescovit: Nuevo registro de Filistatoides insignis para

México

\section{INTRODUCCIÓN}

El término sinantropismo engloba a los organismos que están asociados con los humanos o con sus domicilios (Lawrence, 1989). Cuando el hombre modifica constantemente el entorno ambiental, perturba el hábitat original, pero al mismo tiempo crea nuevos hábitats artificiales, los cuales poseen posibilidades óptimas para el establecimiento de muchas especies, las cuales de otra manera no podrían cohabitar con el ser humano (Tischler, 1973). Las especies sinantrópicas son capaces de adaptarse a la variedad de actividades humanas para asegurar su crecimiento, a menudo extendiendo su distribución natural a otros hábitats como lo son casas, jardines, granjas, edificios, incluso en los basureros (Di Castri et al., 1990). Entre los artrópodos que han podido adaptarse a los ambientes urbanos se encuentran las arañas, ya que algunos aspectos de su biología pueden proveerles de algunas ventajas y desventajas, para realizar la transición de un ambiente natural, hacia uno urbano (Durán-Barrón, 2004).

A nivel mundial, las investigaciones enfocadas con arañas sinantrópicas se han desarrollado en Estados Unidos (Guarisco, 1999), Cuba (Armas, 2003, 2012), Rumania (Urák, 2005), Eslovenia (Kostanjsêk \& Celestina, 2008), Brasil (Brescovit, 2002; Brazil et al., 2005; Melo et al., 2010; Luciane et al., 2011) y Chile (Taucare-Ríos, 2013). En México, las investigaciones realizadas con este grupo, inició con los trabajos de Jiménez (1998), quien estudio la araneofauna asociada a las viviendas en La Paz, B.C.S., y con el estudio de salticidos sinantrópicos realizado por Castelo \& Pérez (1999) en la Ciudad de México. Posteriormente, en esta misma ciudad se desarrollaron otros trabajos (Durán-Barrón, 2004, 2007; Durán-Barrón \& PérezOrtíz, 2005, 2016; Durán-Barrón et al., 2009, 2016; López-Villegas et al., 2012; Salceda-Sánchez et al., 2017). Estudios similares se llevaron a cabo en las ciudades de Puerto Vallarta, Jalisco (Cupul-Magaña \& Navarret-Heredia, 2018); Toluca, Estado de México (Desales-Lara et al., 2011, 2013); Ciudad Victoria, Tamaulipas (Salazar-Olivo \& Solís-Rojas, 2015); Chilpancingo, Guerrero (Rodríguez-Rodríguez et al., 2015); Morelia, Michoacán (Maldonado-Carrizales \& Ponce-Saavedra, 2017; Maldonado-Carrizales et al., 2018) y Mérida, Yucatán (Quijano-Ravell \& Ponce-Saavedra, 2017).

En Chiapas, hasta el momento, no se ha realizado un estudio de esta índole, sin embargo, es uno de los estados en la República Mexicana con un amplio listado de especies de arañas publicado (GarcíaVillafuerte, 2009b; Ibarra-Núñez, 2013). Con el descubrimiento de Filistatoides insignis (O. PickardCambridge, 1896) (Filistatidae), un nuevo registro de araña sinantrópica para México, hallado en la ciudad de Tuxtla Gutiérrez, el objetivo del presente estudio es actualizar el listado de arañas (Araneae) de Chiapas.

\section{MATERIALES Y MÉTODOS}

El material examinado fue recolectado en el Museo de Paleontología "Eliseo Palacios Aguilera" (16 ${ }^{\circ} 45^{\text {' }}$ $35^{\prime}$ N, $93^{\circ} 06^{\prime} 18^{\prime}$ W), ubicado en la zona centro de la ciudad de Tuxtla Gutiérrez, Chiapas, con una elevación de 522 metros, el clima en la ciudad es cálido subhúmedo con lluvias en verano $\mathrm{A}(\mathrm{w})$, con una temperatura media anual máxima de $38.5^{\circ} \mathrm{C}$ y mínima de $14.8^{\circ} \mathrm{C}$, la precipitación promedio anual es de $891.5 \mathrm{~mm}$ (INEGI, 2017). La recolecta de arañas se llevó a cabo durante las dos primeras semanas de septiembre de 2017. La búsqueda de los especímenes fue principalmente visual, mediante la revisión de muebles, contenedores, las paredes y el techo del Museo, entre las 11:00 y 15:00 horas. Los especímenes fueron recolectados manualmente utilizando un pincel y se ubicaron en un tubo vial para ser preservados en alcohol al 80\%. La determinación taxonómica a nivel familia se dio con base a Ubick et al. (2005) y a nivel especifico con el trabajo de Brescovit et al. (2016), con ayuda de un microscopio estereoscópico Zeiss®, modelo Stemi 2000-C. El nuevo registro es ilustrado con fotografías digitales tomadas con una cámara Canon EOS Reflex 6D, adaptada al microscopio estereoscópico y con ayuda del programa Adobe Ilustrator CC 2015. Los ejemplares están depositados en la Colección de Arácnidos de la Universidad de Ciencias y Artes de Chiapas. 
Para la actualización del listado faunístico de arañas del estado de Chiapas, se tomó como punto base los trabajos de García-Villafuerte (2009b) e Ibarra-Núñez (2013) y con base en estos se realizó una búsqueda bibliográfica exhaustiva de las especies citadas para el estado, en el World Spider Catalog (2019). A fin de no repetir información, en este trabajo se consideran únicamente las especies que no fueron incluidas en los listados anteriores de los autores ya mencionados, así como las especies que se reportaron entre el 2010 a 2019 para Chiapas y el nuevo registro aquí descubierto.

\section{RESULTADOS}

\section{Nuevo registro:}

Familia Filistatidae Ausserer 1867

Filistatoides insignis (O. Pickard-Cambridge, 1896). Figuras 1-4.

Diagnosis y descripción: Brescovit et al. (2016).

Material examinado: $3 \hat{\jmath}, 7 q$ y 4 juveniles (CAUNICACH-AR000010). Tuxtla Gutiérrez, Chiapas; México. Museo de Paleontología "Eliseo Palacios Aguilera" (16 45' 35” N, 9306' 18” W). Coutiño José M. A. y García-Villafuerte M. A, cols. Altitud: 522 m.

Historia natural: los especímenes fueron capturados principalmente en recovecos, ubicados en las paredes del área de Servicio del Museo de Paleontología "Eliseo Palacios Aguilera", también fueron capturados de entre cables y dentro de un tubo con diferentes lupas cerca del área de la Colección Paleontológica.

Distribución: Guatemala (World Spider Catalog, 2019). Primer registro para México.

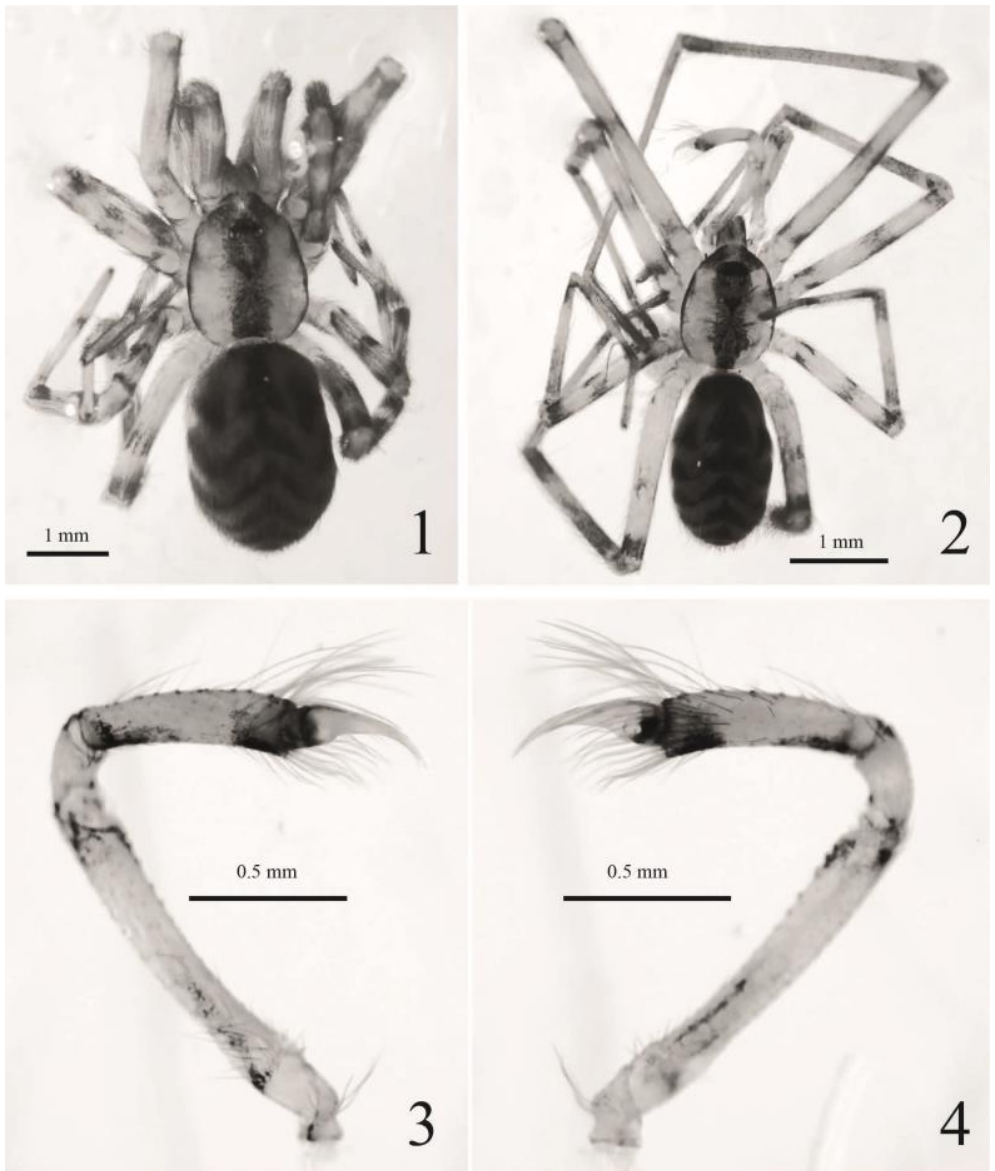

Figuras 1-4. Filistatoides insignis: 1) Habitus dorsal de la hembra, 2) Habitus dorsal del macho, 3) Pedipalpo vista prolateral, 4) Pedipalpo vista retrolateral. 
García-Villafuerte \& Brescovit: Nuevo registro de Filistatoides insignis para

México

\section{Arañas de Chiapas}

Se incluyen 15 especies que no fueron consideradas en los listados anteriores. Al realizar la búsqueda de nuevos registros de arañas para Chiapas, se encontró que del 2010 al 2019 se describieron y reportaron 39 especies más (Ibarra-Núñez et al., 2011; World Spider Catalog, 2019), las cuales son incluidas en el Cuadro 1. Las familias son listadas en orden sistemático siguiendo el modelo del World Spider Catalog (2019). Con base en el nuevo registro y la búsqueda bibliográfica de las especies reportadas para el estado, Chiapas alberga un total de 533 especies, 231 géneros y 53 familias. Las familias con mayor número de especies son Araneidae (106), Theridiidae (105), Salticidae (57) y Linyphiidae (27), en comparación con las familias Miturgidae, Nemesiidae, Oecobiidae, Philodromidae, Plectreuridae, Segestriidae, Senoculidae, Sicariidae, Titanoecidae, Trechaleidae, Zorocratidae y Zoropsidae quienes solo están representadas por una especie.

Cuadro 1. Lista complementaria de especies actuales de arañas (Araneae) registradas para Chiapas. (*) Indica el nuevo registro de Filistatidae reportada para México. (+) Señala las 15 especies que no fueron agregadas en los listados anteriores. (++) Señala las 39 especies reportadas entre 2010 y 2019 de acuerdo con Ibarra-Núñez et al. (2011) y World Spider Catalog (2019).

\begin{tabular}{|c|c|c|}
\hline Familia & Género & Especie \\
\hline \multirow{5}{*}{ Agelenidae } & Eratigena & florea Brignoli, $1974(+)$ \\
\hline & Melpomene & transversa (F. O. Pickard-Cambridge, 1902) (++) \\
\hline & Novalena & shlomitae García-Villafuerte, 2009a (+) \\
\hline & Novalena & simplex (F.O. Pickard-Cambridge 1902) $(++)$ \\
\hline & Rualena & $\begin{array}{l}\text { pasquini Brignoli, } 1974 \text { (puede estar fuera de lugar, por Maya-Morales y } \\
\text { Jiménez, 2016) (+) }\end{array}$ \\
\hline Anyphaenidae & Josa & nigrifrons (Simon, 1897) (++) \\
\hline \multirow{2}{*}{ Araneidae } & Mangora & ixtapan Levi, 2005(++) \\
\hline & Taczanowskia & gustavoi Ibarra-Núñez, 2013 (++) \\
\hline \multirow{3}{*}{ Clubionidae } & Elaver & helenae Saturnino \& Bonaldo, 2015 (++) \\
\hline & Elaver & candelaria Saturnino \& Bonaldo, $2015(++)$ \\
\hline & Elaver & mirabilis (O. Pickard-Cambridge, 1896) $(++)$ \\
\hline \multirow{2}{*}{ Ctenizidae } & Ummidia & zebrina (F. O. P.-Cambridge, 1897) (++) \\
\hline & Ummidia & zilchi Kraus, $1955(++)$ \\
\hline Dipluridae & Euagrus & pristinus O. P.- Cambridge, 1899 (+) \\
\hline \multirow{2}{*}{ Filistatidae } & Filistatoides & insignis (O. Pickard-Cambridge, 1896) (*) \\
\hline & Kukulkania & santosi Magalhaes \& Ramírez, 2019(++) \\
\hline Leptonetidae & Darkoneta & arganoi Brignoli, $1974(+)$ \\
\hline \multirow{5}{*}{ Linyphiidae } & Grammonota & teresta Chikering, $1970(++)$ \\
\hline & Linyphia & nigrita (F. O. P.-Cambridge, 1902) (++) \\
\hline & Mermessus & trilobatus (Emerton, 1882) (++) \\
\hline & Selenyphantes & longispinosus (O. P.-Cambridge, 1896) (++) \\
\hline & Tennesseellum & formica (Emerton, 1882) $(++)$ \\
\hline Mysmenidae & Maymena & sbordonii Brignoli, $1974(+)$ \\
\hline \multirow{3}{*}{ Ochyroceratidae } & Ochyrocera & chiapas Valdez-Mondragón, 2009 (+) \\
\hline & Ochyrocera & fagei Brignoli, $1974(+)$ \\
\hline & Ochyrocera & pojoj Valdez-Mondragón, 2017 (++) \\
\hline \multirow{9}{*}{ Oonopidae } & Dysderina & plena O. P.-Cambridge $1894(++)$ \\
\hline & Emboonops & arriaga Bolzerm, Platnick \&Berniker, $2015(++)$ \\
\hline & Emboonops & bonampak Bolzerm, Platnick \&Berniker, $2015(++)$ \\
\hline & Emboonops & calco Bolzerm, Platnick \&Berniker, $2015(++)$ \\
\hline & Emboonops & palenque Bolzerm, Platnick \&Berniker, $2015(++)$ \\
\hline & Guatemoonops & rhino Bolzerm, Platnick \&Berniker, $2015(++)$ \\
\hline & Oonopoides & zullinii Brignoli, $1974(+)$ \\
\hline & Scaphiella & cocona Platnick \&Dupérré, 2010 (+) \\
\hline & Toloonops & chiapa Bolzerm, Platnick \&Berniker, 2015 (++) \\
\hline
\end{tabular}




\begin{tabular}{|c|c|c|}
\hline Familia & Género & Especie \\
\hline & Toloonops & chickeringi (Brignoli, 1974) (+) \\
\hline \multirow{3}{*}{ Pholcidae } & Ixchela & santibanezi Valdez-Mondragón 2013 (++) \\
\hline & Ixchela & tzotzil Valdez-Mondragón 2013 (++) \\
\hline & Modisimus & deltoroi Valdez-Mondragón \&Fracke, 2009 (+) \\
\hline \multirow{2}{*}{ Phrurolithidae } & Phonotimpus & pennimani Chamé-Vázquez, Ibarra-Núñez \& Jiménez, 2018 (++) \\
\hline & Phonotimpus & talquian Chamé-Vázquez, Ibarra-Nuñez \& Jiménez, 2018 (++) \\
\hline \multirow{2}{*}{ Salticidae } & Freya & regia (Peckham \& Peckham, 1896) (+) \\
\hline & Tarkas & maculatipes (F. O. Pickard-Cambridge, 1901) (+) \\
\hline \multirow{2}{*}{ Tetragnathidae } & Leucage & acuminata (O. P.-Cambridge, 1889) (++) \\
\hline & Leucauge & simplex F. O. P.-Cambridge, $1903(++)$ \\
\hline Theraphosidae & $\begin{array}{l}\text { Citharacanthus } \\
\text { Hemirraghus }\end{array}$ & $\begin{array}{l}\text { alvarezi Estrada-Álvarez, Guadarrama \& Martínez, 2013(++) } \\
\text { perezmilesi García-Villafuerte \&Locht } 2010(+)\end{array}$ \\
\hline \multirow{4}{*}{ Theridiidae } & Phycosoma & altum (Keyserling, 1886) (++) \\
\hline & Thymoites & puer (Mello-Leitão, 1941) (++) \\
\hline & Wamba & congener O. P.-Cambridge, $1896(++)$ \\
\hline & Wirada & mexicana Campuzano \& Ibarra-Núñez (2018) (++) \\
\hline Thomisidae & $\begin{array}{l}\text { Misumenoides } \\
\text { Tmarus }\end{array}$ & $\begin{array}{l}\text { annulipes (O. P.-Cambridge, 1891) (++) } \\
\text { vitusus } \text { Chickering, } 1965(++)\end{array}$ \\
\hline Uloboridae & Philoponella & tingens (Chamberlin \&Ivie, 1936) (++) \\
\hline Zoropsidae & Tengella & kalebi Candia-Ramírez \& Valdez-Mondragón 2013 (++) \\
\hline
\end{tabular}

\section{DISCUSIÓN}

El género Filistatoides se registra, hasta el momento, únicamente en América con distribución de México a Chile (World Spider Catalog, 2019). En este estudio, se amplía la distribución geográfica de Filistatoides insignis hacia la región sureste de México, como parte de la araneofauna sinantrópica de Chiapas, pues únicamente se citaba para Guatemala en la región litoral de Iztapa y asociadas a edificios abandonados (Brescovit et al., 2016).

En relación con las especies sinantrópicas del Orden Araneae en Chiapas, hasta el momento no se ha realizado un trabajo de investigación formal y sistematizado. El descubrimiento de $F$. insignis, nos invita a reflexionar sobre la importancia de este tipo de investigaciones, para tener un conocimiento más integral de la riqueza y diversidad de estas arañas en el estado.

Armas (2012) menciona que las especies sinantrópicas revisten gran interés por su estrecha asociación con el hombre y las áreas donde este vive o desarrolla sus actividades diarias, fenómeno que adquiere mayor relevancia cuando se tratan de organismos que, de un modo u otro, pueden afectar la salud humana o incidir en el desarrollo de alguna actividad socioeconómica. Por lo tanto, es importante llevar a cabo trabajos multidisciplinarios entre aracnólogos, médicos y la población en general, para tener un mejor entendimiento de este grupo de arácnidos. En este caso, $F$. insignis, no representan peligro alguno para los humanos ni para las actividades económicas, tal vez pueda amenazar a las especies nativas ocupando sus espacios, si se propagara fuera de las áreas urbanas.

\section{Actualización del listado araneofaunistico para Chiapas}

En su relación bibliográfica preliminar de arañas para México, Hoffman (1976), reporta 169 especies para Chiapas. Posteriormente, Jiménez (1996) menciona que la araneofuna chiapaneca se componía de 281 especies. Años más tarde, García-Villafuerte (2009b) elabora un listado de arañas, enumerando 377 especies y 87 morfoespecies a nivel género. Por su parte, Ibarra-Núñez (2013) aportó para Chiapas un nuevo listado en donde enumera 478 especies y siete morfoespecies aun por describir. Ibarra-Núñez (com. pers., 2017) señala que en su investigación registró las especies de arañas válidamente publicadas para Chiapas hasta 
García-Villafuerte \& Brescovit: Nuevo registro de Filistatoides insignis para

México

2009. Sin embargo, Ibarra-Núñez (2013) no consideró 15 especies registradas para el estado (Brignoli, 1974; Galiano, 2001; García-Villafuerte, 2009a; Valdez-Mondragón, 2009). Estos 15 registros se complementan con las 21 especies reportadas por Ibarra-Núñez et al. (2011) y la búsqueda exhaustiva en el World Spider Catalog (2019), en donde se reportan 18 especies de arañas para la entidad publicadas entre el 2010 y 2019. Considerando lo antes mencionado, e incluyendo el nuevo registro reportado en este estudio para México, se tiene un total de 533 especies de arañas para el estado de Chiapas validadas a la fecha.

\section{CONCLUSIONES}

Considerando los datos de Duran-Barrón et al. (2016) y Desales-Lara et al. (2018), Chiapas alberga el 22.2\% de la biodiversidad de arañas a nivel Nacional. Este cambio ubica al estado en el primer lugar de mayor riqueza de especies de arañas en el país. En este contexto y a pesar de que se han registrado 2,392 especies para el país, el Orden Araneae es uno de los grupos menos estudiados en México (Duran-Barrón et al., 2016; Desales-Lara et al., 2018). Consecuentemente, es muy importante que se continúe con la realización de estudios araneofaunisticos sistematizados en ambientes naturales, así como la implementación de técnicas para estudiar sistematizadamente la araneofauna urbana en Chiapas, de tal manera que podamos tener un conocimiento integral no solo de la fauna de arañas y como están asociadas a la composición y estructura de la vegetación y sus microhábitats, sino también de su composición en ambientes urbanos y suburbanos.

AgRadecimientos. Estamos agradecidos con Marco Antonio Coutiño, por haber encontrado el primer ejemplar de Filistatoides insignis. A Fabián Salgado Álvarez (Nitido Studio) por gentilmente tomar las fotografías. A Guillermo Ibarra-Núñez (El Colegio de la Frontera Sur, Unidad Tapachula) por proporcionarnos su listado de arañas para Chiapas y los acertados comentarios y correcciones al manuscrito. De igual manera, a cada uno de los revisores anónimos, por sus valiosos e importantes comentarios y sugerencias al manuscrito. Este trabajo se lleva a cabo como complemento de las investigaciones realizadas por MAG-V dentro del proyecto "Prospección y Resguardo del Patrimonio Paleontológico de Chiapas", generosamente financiado por el Gobierno del Estado de Chiapas. Para CNPq processo 301776/2004-0 para ADB. A la memória de los hermamos Abí Umorí Sergio y Ezequiel García Reyes.

\section{LITERATURA CITADA}

Armas, L. F. (2003) Notas sobre los arácnidos de mi casa (Chelicerata Arachnida). Revista Ibérica de Aracnología, 8, 143-149.

Armas, L. F. (2012) Arácnidos sinántropicos de Cuba (Arachnida excepto Acari). Boletín de la Sociedad Entomológica Aragonesa, 50, 513-516.

Brazil, T. K., Almeida-Silva, L. M., Pinto-Leite, C. M., Lira-da-Silva, R. M., Lima, P. M. C., Brescovit, A. D. (2005) Aranhas sinántropicas em três bairros de cidade de Salvador, Bahia, Brasil (Arachnida, Araneae). Biota Neotropica, 5, 1-7.

Brescovit, A. D. (2002) Arahas, espécies sinantrópicas, accidentes e controle. Saúde Ambienal, 49, $24-27$.

Brescovit, A. D., Sánchez-Ruiz, A., Alayón G. G. (2016) The Filistatidae in the Caribbean region, with a description of the new genus Antilloides, revision of the genus Filistatoides F. O. P.-Cambridge and notes on Kukulcania Lehtinen (Arachnida, Araneae). Zootaxa, 4136, 401-432.

Brignoli, P. M. (1974) Notes on spiders, mainly cave-dwelling, of southern Mexico and Guatemala (Araneae). Quaderno, Accademia Nazionale dei Lincei, 171 (2), 195-238.

Castelo, J. L., Pérez, T. M. (1999) El problema en la determinación taxonómica de las arañas Salticidae (Arachnida, Araneae) habitantes de viviendas de la Ciudad de México. Memorias del XXXIV Congreso Nacional de Entomología. México. 33-37 pp. 
Cupul-Magaña, F. G., Navarrete-Heredia, J. L. (2008) Artropofauna de las viviendas de Puerto Vallarta, Jalisco, México. Ecología Aplicada, 7, 187-190.

Desales-Lara, M. A., Francke, O. F., Sánchez-Nava, P. (2011) Arañas (Arachnida, Araneae) asociadas a diferentes grados de urbanización. Memorias del XLVI Congreso Nacional de Entomología. México. 69-73 pp.

Desales-Lara, M. A., Francke, O. F., Sánchez-Nava, P. (2013) Diversidad de arañas en hábitats antropogénicos. Revista Mexicana de Biodiversidad, 84 (1), 291-305.

Di Castri, F., Hansen, A. J., Debussche, M. (1990) Biological invasions in Europe and the Mediterranean Basin. Kluwer Academic Publishers. USA, Springer, 458 pp.

Durán-Barrón, C. G. (2004) Diversidad de arañas (Arachnida: Araneae) asociadas a las viviendas de la Ciudad de México (Área metropolitana) (tesis de maestría). Facultad de Ciencias, Universidad Autónoma de México, D. F., México.

Durán-Barrón, C. G. (2007) El sinantrópismo en arañas. Memorias del VI Congreso Latinoamericano de Entomologia y XLII Congreso Nacional de Entomología. México. 9-13 pp.

Durán-Barrón., C. G., Pérez-Ortíz, T. M. (2005) Diversidad de familias de arañas asociadas a viviendas humanas de la Ciudad de México. Memorias del XL Congreso Nacional de Entomología. Sociedad Mexicana de Entomología. México. 66-70 pp.

Durán-Barrón, C. G., Pérez-Ortíz, T. M. (2016) Arañas de importancia médica: la viuda negra y la araña violinista, pp. 239-244. In: CONABIO/SEDEMA (Eds). La biodiversidad de la Ciudad de México, Vol. II. Comisión Nacional para el Conocimiento y Uso de la Biodiversidad (CONABIO) y Secretaría del Medio Ambiente del Distrito Federal (SEDEMA), México.

Durán-Barrón., C. G., Francke, O. F., Pérez-Ortiz, T. M. (2009) Diversidad de arañas (Arachnida: Araneae) asociadas a viviendas de la Ciudad de México (Área metropolitana). Revista Mexicana de Biodiversidad, 80, 55-69.

Durán-Barrón, C. G., Montiel-Parra, G., Valdez-Mondragón, A., Villegas-Guzmán, G. A., ParedesLeón, R., Pérez, T. M. (2016) Arácnidos (Arachnida), pp. 229-238. In: CONABIO/SEDEMA (Eds). La biodiversidad de la Ciudad de México, Vol. II. Comisión Nacional para el Conocimiento y Uso de la Biodiversidad (CONABIO) y Secretaría del Medio Ambiente del Distrito Federal (SEDEMA), México.

Galiano, M. E. (2001) Revisión de las especies de Freya del grupo decorata (Araneae, Salticidae). Journal of Arachnology, 29, 21-41.

García-Villafuerte, M. A. (2009a) A new species of Rualena (Araneae, Agelenidae) from Chiapas, Mexico. Revista Ibérica de Aracnología, 17, 7-11.

García-Villafuerte, M. A. (2009b) La araneofauna (Araneae) reciente y fósil de Chiapas, México. Revista Mexicana de Biodiversidad, 80, 633-646.

Guarisco, H. (1999) House spiders of Kansas. Journal of Arachnology, 27, 217-221.

Hoffmann, A. (1976) Relación bibliográfica preliminar de las arañas de México (Arachnida: Araneae). Universidad Nacional Autónoma de México (Ed). Instituto de Biología, México.

Ibarra-Núñez, G. (2013) Diversidad de las arañas (Arachnida: Araneae), pp. 191-196. In: Cruz-Angón, A., Melgarejo, E. D., Camacho-Rico, F., Nájera-Cordero, K. C. (Eds.). La biodiversidad en Chiapas, México. Comisión Nacional para el Conocimiento y Uso de la Biodiversidad/ Gobierno del Estado de Chiapas, Vol. 2, México.

Ibarra-Núñez, G., Maya-Morales, J., Chamé-Vázquez, D. (2011) Las arañas del bosque mesófilo de montaña de la Reserva de la Biosfera Volcán Tacaná, Chiapas, México. Revista Mexicana de Biodiversidad, 82, 1183-1193.

INEGI (2017) Anuario estadístico y geográfico de Chiapas 2017. Disponible en: https://www.datatur.sectur.gob.mx/ITxEF_Docs/CHIS_ANUARIO_PDF.pdf (Fecha de acceso abril 2017).

Jiménez, M. L. (1996) Araneae, pp. 83-101. In: Llorente-Bousquets, J., García Aldrete, A. N., GonzálezSoriano (Eds.). Biodiversidad, Taxonomía y Biogeografía de Artrópodos de México: Hacia una síntesis de su conocimiento. Instituto de Biología, UNAM, México, DF. 
García-Villafuerte \& Brescovit: Nuevo registro de Filistatoides insignis para

México

Jiménez, M. L. (1998) Aracnofauna asociada a las viviendas de la ciudad de La Paz, Baja California Sur, México. Folia Entomológica Mexicana, 102, 1-10.

Kostanjsêk, R., Celestina, A. (2008) New records of synanthropic spider species (Arachnida, Araneae) in Slovenia. Natura Slovniae, 10, 51-55.

Lawrence, E. (1989) Henderson's dictionary of biological terms. Willey Interscience, New York, 704 pp.

López-Villegas, V. M. S., Castelo-Calvillo, J. L., Víctor-Rosas, L. J. (2012) Listado faunístico de arañas de la familia Salticidae (Aracnida: Araneae) del Distrito Federal, México. Entomología Mexicana, $11,59-62$.

Luciane F, M., Brandes G, C., Bazílio, S., Ricetti, J. (2011) Araneofauna sinantrópica associada com a família Sicariidae no município de União da Vitória, Paraná, Brasil. Sitientibus série Ciências Biológicas, 11, 48-56.

Maldonado-Carrizales, J., Ponce-Saavedra, J. (2017) Arañas Saltarinas (Araneae: Salticidae) en dos sitios contrastantes en grado de antropización en Morelia Michoacán, México. Entomología mexicana, 4, 597-603.

Maldonado-Carrizales, J., Quijano-Ravell, A., Guzmán-García, C. E., Ponce-Saavedra, J. (2018) Arañas (Araneae: Araneomorphae) antrópicas de Morelia, Michoacán, México. Entomología Mexicana, 5, 22-28.

Maya-Morales, J., Jiménez, M. L. (2016) Taxonomic revision of the spider genus Rualena Chamberlin \& Ivie 1942 and description of Hoffmannilena, a new genus from Mexico (Araneae: Agelenidae). Zootaxa, 4084 (1), 1-49.

http://10.11646/zootaxa.4084.1.1

Melo, T. S., Peres, M. C. L., Dias, M. A., Benati, K. R., Andrade, A. R. S., Guimãrae, M. V. A. (2010) Metropolitan spider fauna in the Brazilian Atlantic Forest remnants under urban pressure. Abstracts of 18th International Congress of Arachnology. Poland. 290-292 pp.

Quijano-Ravell, A., Ponce-Saavedra, J. (2017) Géneros de salticídos urbanos (Araneae: Salticidae) de Ciudad Caucel, Comisaría de Mérida, Yucatán, México. Entomología mexicana, 4, 604-609.

Rodríguez-Rodríguez, S. E., Solís-Catalán, K. P., Valdez-Mondragón, A. (2015) Diversity and seasonal abundance of anthropogenic spiders (Arachnida: Araneae) in different urban zones of the city of Chilpancingo, Guerrero, Mexico. Revista Mexicana de Biodiversidad, 86, 962-971.

Salceda-Sánchez, B., Hernández-Hernández, V., Conde-Sánchez, E., Vargas-Olmos, M., LópezCárdenas, J., Huerta, H. (2017) Nuevos registros de distribución del género Latrodectus Walckenaer y Loxosceles Heineken y Lowe en México. Southwestern Entomologist, 42, 575-582. http://doi.org/10.3958/059.042.0226

Salazar-Olivo, C. A., Solís-Rojas, C. (2015) Araneofauna urbana (Arachnida: Araneae) de Ciudad Victoria, Tamaulipas, México. Acta Zoológica Mexicana, 31, 55-66.

Taucare-Ríos, A. (2013) Las arañas sinantrópicas peligrosas de Chile. Revista Médica de Chile, 140, 12281229.

Tischler, W. (1973) Ecology of arthropod fauna in man-made hábitats: The problema of synanthropid. Zoologische Anzeiger, 191, 157-161.

Ubick, D., Paquin, P., Cushing, P., Dupérré, N. (2005) Spiders of North America: an identification manual. American Arachnological Society, EUA, 377 pp.

Urák, I. (2005) Two new invasive alien spider (Arachnida:Araneae) in Romanian Arachnofauna. Entomologica Romanica, 10, 89-91.

Valdez-Mondragón, A. (2009). Two new species of the spider genus Ochyrocera (Araneae, Ochyroceratidae) from Mexico. Journal of Arachnology, 37, 170-177.

World Spider Catalog. (2019) World Spider Catalog, Naural History Museum Bern. Disponible en: http://wsc.nmbe.ch, version 18.5 (Fecha de acceso marzo 2019). 